\title{
ON THE EXTREME EIGENVALUES OF TRUNCATED TOEPLITZ MATRICES
}

\author{
BY SEYMOUR V. PARTER
}

Communicated by Edwin Moise, December 23, 1960

Let $f(\theta)$ be a real-valued Lesbesgue integrable function defined on $[-\pi, \pi]$. Let $\left\{C_{j}\right\}$ be the Fourier coefficients of $f(\theta)$, i.e.,

$$
f(\theta) \sim \sum_{-\infty}^{\infty} C_{j} e^{i j \theta}
$$

The matrix $T_{n}[f]=\left(C_{s-j}\right) ; s, j=0,1, \cdots, n$ is the $n$th finite section of the infinite Toeplitz matrix $\left(C_{\varepsilon-j}\right)$ associated with the function $f(\theta)$.

In this note we are concerned with functions $f(\theta)$ satisfying

Condition A. Let $f(\theta)$ be real, continuous and periodic with period $2 \pi$. Let $\min f(\theta)=f(0)=m$ and let $\theta=0$ be the only value of $\theta(\bmod 2 \pi)$ for which this minimum is attained.

Condition $\mathrm{A}(\alpha)$. Let $f(\theta)$ be a function satisfying condition $A$. Moreover, let $f(\theta)$ have continuous derivatives of order $2 \alpha$ in some neighborhood of $\theta=0$. Finally let $f^{(2 \alpha)}(0)=\sigma^{2}>0$ be the first nonvanishing derivative of $f(\theta)$ at $\theta=0$.

TheOREM. Let $f(\theta)$ satisfy conditions $A$ and $A(\alpha)$. Let $\lambda_{r, n}(\nu=1,2, \cdots, n+1)$ be the eigenvalues of $T_{n}[f]$ arranged in nondecreasing order. For fixed $\nu$, as $n \rightarrow \infty$ we have

$$
\lambda_{\nu, n}=m+\frac{\sigma^{2}}{(2 \alpha) !} \Lambda_{\nu}\left(\frac{1}{n}\right)^{2 \alpha}+o\left(\frac{1}{n}\right)^{2 \alpha},
$$

where the numbers $\Lambda_{\nu}$ are the eigenvalues arranged in nondecreasing order of

$$
\left[-\left(\frac{d}{d x}\right)^{2}\right]^{\alpha} U-\Lambda U=0, \quad 0 \leqq x \leqq 1,
$$

with boundary conditions

$$
\left(\frac{d}{d x}\right)^{i} U(0)=\left(\frac{d}{d x}\right)^{i} U(1)=0, \quad i=0,1, \cdots, \alpha-1 .
$$

The case $\alpha=1$ was studied by Kac, Murdock and Szegö [3]. In [5] Widom also studied the case $\alpha=1$ and, under suitable conditions, obtained the next term in the asymptotic expansion of $\lambda_{\nu, n}$. The case $\alpha=2$ was studied by this author [4]. 
The validity of this theorem was conjectured by Widom [6]. In fact, his conjecture is much more general.

The author is indebted to Professors Kac and Widom for many fruitful discussions concerning these problems.

In view of the Weyl-Courant characterization of $\lambda_{\nu, n}$ (and $\Lambda_{\nu}$ ) as solutions of a variational problem, it is sufficient to consider the case where $f(\theta)$ is an even trigonometric polynomial. (See [4] or [5] for a more detailed argument.) Moreover, there is no loss in generality in assuming $m=0$. Thus $f(\theta)$ may be written as

$$
f(\theta)=\beta_{0}(1-\cos \theta)^{\alpha}+\sum_{k=1}^{N} \beta_{k}(1-\cos \theta)^{k+\alpha}
$$

where

$$
\beta_{0}=\frac{2^{\alpha} \sigma^{2}}{(2 \alpha) !}
$$

Let us interpret the eigenvalue problem as a difference equation. Let $R=N+\alpha-1$ and let $D_{n}$ be the interval $[-R /(n+2), 1+R /(n+2)]$. Let $\Delta x=1 /(n+2)$ and let $x_{j}=j \Delta x$ be the lattice points in $D_{n}$. If $\phi(x)$ is any function defined on $D_{n}$ we denote $\phi\left(x_{j}\right)$ by $\phi_{j}$.

Let $P_{n}$ be the class of piecewise-linear functions $h(x)$ defined on $D_{n}$ and determined by their values at $x_{j}$ which satisfy

$$
h_{j}=0 \text { for } j \leqq 0 \text { and } \geqq n+2 \text {. }
$$

Let

$$
T_{n}\left[(1-\cos \theta)^{r}\right]=\tau_{r}
$$

and let $\delta$ be the second central divided difference operator, i.e.,

$$
(\delta \phi)_{j}=\left(\frac{1}{\Delta x}\right)^{2}\left\{\phi_{j+1}-2 \phi_{j}+\phi_{j-1}\right\} .
$$

We observe that every function $h(x) \in P_{n}$ corresponds to an $(n+1)$ vector $H=\left(h_{j}\right), j=1,2, \cdots, n+1$, and conversely.

Furthermore, it is easy to relate the matrices $\tau_{r}(r \leqq R+1)$ to the operator $\delta$. We have

$$
\left(\tau_{r} H\right)_{j}=\left(-\frac{1}{2} \Delta x^{2}\right)^{r}\left(\delta^{r} h\right)_{j}, \quad=1,2, \cdots n+1
$$

thus 
(4.4) $\quad\left(T_{n}[f] H\right)_{j}=\beta_{0}\left(-\frac{1}{2} \Delta x^{2}\right)^{\alpha}\left(\delta^{\alpha} h\right)_{j}+\sum_{k=1}^{N}\left(-\frac{1}{2} \Delta x^{2}\right)^{\alpha+k} \beta_{k}\left(\delta^{\alpha+k} h\right)_{j}$.

Let $S_{n}$ be the finite difference operator which corresponds to $(n+2)^{2 \alpha} T_{n}[f]$, i.e.,

$$
S_{n}=\left(-\frac{1}{2}\right)^{\alpha} \beta_{0} \delta^{\alpha}+\sum_{k=0}^{N}\left(-\frac{1}{2} \Delta x^{2}\right)^{k} \beta_{k} \delta^{\alpha+k} .
$$

Clearly, $S_{n}$ is a consistent approximation to the differential operator

$$
\frac{\sigma^{2}}{(2 \alpha) !}\left[-\left(\frac{d}{d x}\right)^{2}\right]^{\alpha}
$$

Thus our theorem is seen to be equivalent to the theorem that the eigenvalues $\Lambda_{\nu, n}$ of $S_{n}$ acting on functions $h(x) \in P_{n}$ converge to the eigenvalues of (4.5a) subject to the boundary conditions (2a).

We require one more definition. Let $h(x), g(x) \in P_{n}$, let $H$ and $G$ be the corresponding $(n+1)$ vectors, then

$$
[h, g] \equiv \Delta x \sum h_{j} g_{j}=\Delta x(H, G) .
$$

Lemma 1.

$$
\operatorname{LimSup}_{n \rightarrow \infty} \Lambda_{\nu, n}=\operatorname{LimSup}_{n \rightarrow \infty}(n+2)^{2 \alpha} \lambda_{\nu, n} \leqq \frac{\sigma^{2}}{(2 \alpha) !} \Lambda_{\nu} .
$$

Proof. This follows immediately from the Weyl-Courant characterization of $\lambda_{\nu, n}$ and the appropriate choice of "test" vectors obtained from the eigenfunctions of (2). (See Weinberger [7] where this is carried out in detail for a similar problem.)

Let $\Delta(\alpha)$ be the divided-difference operator of order $\alpha$ determined as follows:

$$
\alpha=2 \gamma: \Delta(\alpha)=\delta^{\gamma}
$$

and

$$
\alpha=2 \gamma+1: \Delta(\alpha)=\delta^{\gamma} \cdot D
$$

where $D$ is a first order divided-difference operator (forward or backward, it doesn't matter).

Lemma 2. Let $H$ be an eigenvector of $T_{n}[f]$ associated with $\lambda_{\nu, n}$ and let $h(x) \in P_{n}$ be the associated function with $h(x)$ (i.e., $\left.H\right)$ normalized so that $[h, h]=1$.

There exists a constant $M_{\nu}$ independent of $n$, such that 


$$
[\Delta(\alpha) h, \Delta(\alpha) h] \leqq M_{\nu}
$$

Proof. We first prove that

$$
\left[(-\delta)^{\alpha} h, h\right] \leqq M_{\nu},
$$

and (5.2) follows from $\alpha$ applications of summation by parts. (Note: $-\delta$ is a positive definite operator.)

However, (5.2a) is equivalent to

$$
\Delta x \cdot 2^{\alpha}(n+2)^{2 \alpha}\left(\tau_{\alpha} H, H\right) \leqq M_{\nu} .
$$

Now, Lemma 1 implies the existence of a constant $L_{\nu}$ such that

$$
\Delta x(n+2)^{2 \alpha}\left(T_{n}[f] H, H\right) \leqq L_{\nu} .
$$

However, as is well known (see [4] or [5]), if $\phi(\theta)=\sum_{j=1}^{n+1} h_{j} e^{i(j-1) \theta}$, then

$$
\left(T_{n}[f] H, H\right)=\frac{1}{2 \pi} \int_{-\pi}^{\pi} f(\theta)|\phi|^{2} d \theta
$$

and

$$
\left(\tau_{\alpha} H, H\right)=\frac{1}{2 \pi} \int_{-\pi}^{\pi}(1-\cos \theta)^{\alpha}|\phi|^{2} d \theta .
$$

We write $f(\theta)$ as $f(\theta)=(1-\cos \theta)^{\alpha} Q(\theta)$, where

$$
Q(\theta)=\beta_{0}+\sum_{k=1}^{N} \beta_{k}(1-\cos \theta)^{k} .
$$

Since $f(\theta)$ satisfies conditions $A$ and $A(\alpha)$, there is a positive constant $Q_{0}$ such that

$$
0<Q_{0} \leqq Q(\theta) .
$$

Thus, (5.3a), (5.3b) together with (5.3) implies

$$
2^{\alpha} \Delta x(n+2)^{2 \alpha}\left(\tau_{\alpha} H, H\right) \leqq 2^{\alpha} \cdot L_{\nu} / Q_{0},
$$

which proves the lemma.

Using Lemma 2 and more-or-less standard techniques in Analysis (see Courant, Friedrichs and Lewy [1]) one readily obtains the following result on the compactness of the eigenfunctions $h(x) \in P_{n}$.

LEMMA 3. Let $\left\{H_{v, n}\right\}$ be a sequence of eigenvectors of $T_{n}[f]$ associated with $\lambda_{r, n}$. Let $H \equiv\left\{h_{n}(x)\right\}$ be the associated sequence of functions in $P_{n}$. There exists a subsequence $\left\{h_{n^{\prime}}(x)\right\}$ which converges uniformly on $[0,1]$ to a function $u(x)$. In addition, $u(x)$ has $(\alpha-1)$ continuous derivatives 
and has strong derivatives of order $\alpha$ which satisfy

$$
\int_{0}^{1}\left|u^{(\alpha)}\right|^{2} d x \leqq M_{\nu}
$$

Moreover, the divided-difference of $h_{n^{\prime}}(x)$ of order $k$ with $k \leqq \alpha-1$ also converge uniformly to the kth derivative of $u(x)$. Finally, in virtue of this last statement

$$
u^{(k)}(0)=u^{(k)}(1)=0, \quad k=0,1,2, \cdots, \alpha-1 .
$$

Our proof is almost complete. Let $\phi(x)$ be any function in $C_{\infty}[0,1]$ which satisfies the boundary conditions (2a). We may extend $\phi$ as a $C_{\infty}$ function in $D_{n}$. There is no confusion if we also call this extended function $\phi$. Also, given such a function $\phi(x)$ we may construct a function $\hat{\phi} \in P_{n}$ in the obvious way.

Consider the sequence $H=\left\{h_{n}(x)\right\}$ associated with $\lambda_{\nu, n}$. We may. choose a subsequence $\left\{h_{n^{\prime}}(x)\right\}$ so that $\Lambda_{\nu, n^{\prime}}=\left(n^{\prime}+2\right)^{2 \alpha} \lambda_{\nu, n^{\prime}}$ converge to a value $\Lambda_{\nu}^{\mathbf{0}}$. We may now choose a subsequence (in accordance with Lemma 3 ) so that the $h_{n^{\prime \prime}}(x) \rightarrow u(x)$. We write $n$ for $n^{\prime \prime}$, and proceed.

LEMmA 4. Let $\phi \in C_{\infty}[0,1]$, then

$$
\left[S_{n} h_{n}, \hat{\phi}\right]=\frac{\sigma^{2}}{\alpha !}(-1)^{\alpha} \int_{0}^{1} u(x)\left(\frac{d}{d x}\right)^{2 \alpha} \phi \cdot d x+o(1) .
$$

Proof. Let $\Phi$ be the $(n+1)$ vector associated with $\hat{\phi}$, then, since $T_{n}[f]$ is hermitian,

$$
\begin{aligned}
{\left[S_{n} h_{n}, \phi\right] } & =\Delta x(n+2)^{2 \alpha}\left(T_{n}[f] H_{n}, \Phi\right) \\
& =\Delta x(n+2)^{2 \alpha}\left(H_{n}, T_{n}[f] \Phi\right) .
\end{aligned}
$$

For any point $x_{j}$ for which $R+1<j<(n+2)-(R+1)$, Taylor's theorem gives us

$$
(n+2)^{2 \alpha}\left(T_{n}[f] \Phi\right)_{j}=\frac{\sigma^{2}}{\alpha !}(-1)^{\alpha}\left(\frac{d}{d x}\right)^{2 \alpha} \phi+O\left(\Delta x^{2}\right)
$$

Consider now any other point $x_{j}, 1 \leqq j \leqq n+1$. Let $\alpha \leqq r \leqq R+1$, then

$$
\begin{aligned}
(n+2)^{2 \alpha}\left(\tau_{r} \Phi\right)_{j}= & \left(-\frac{1}{2}\right)^{r}(\Delta x)^{2(r-\alpha)}\left[\left(\frac{d}{d x}\right)^{2 r} \phi\right]_{j} \\
& +O\left[\phi_{j}\left(\frac{1}{\Delta x}\right)^{2 \alpha}\right] .
\end{aligned}
$$


Since $\phi_{j}=O\left(\Delta x^{\alpha}\right)$, the error term in (5.5b) is $O\left[(1 / \Delta x)^{\alpha}\right]$. Since $h_{j}=o\left(\Delta x^{\alpha-1}\right)$ we find the error in the contribution to 5.4, i.e., the error in

$$
\Delta x(n+2)^{2 \alpha} h_{j}\left(\sigma_{r} \Phi\right)_{j},
$$

is $o(1)$. Thus our lemma is proven.

However, we also have

$$
\left[S_{n} h_{n}, \phi\right] \rightarrow \Lambda_{\nu}^{0} \int_{0}^{1} u(x) \phi(x) d x
$$

which, together with Lemma 4 implies that $u(x)$ is a "weak" eigenfunction (with eigenvalue $\Lambda_{\nu}^{0}$ ) of the operator (4.5a). But, upon considering the equivalent integral equation (using the Green's function), we see that such a weak eigenfunction is indeed an eigenfunction with eigenvalue $\Lambda_{\nu}^{0}$.

However, Lemma 1 and the Weyl-Courant lemma, and the uniqueness of the eigenvalues of (4.5a) show

$$
\Lambda_{\nu}^{0}=\frac{\sigma^{2}}{(2 \alpha) !} \Lambda_{\nu}
$$

\section{REFERENCES}

1. R. Courant, K. O. Friedrichs and H. Lewy, Über die partiellen Differenzgleichungen der mathematisch Physik, Math. Ann. vol. 100 (1928) pp. 32-74.

2. U. Grenander and G. Szegö, Toeplitz forms and their applications, Berkeley, University of California Press, 1958.

3. M. Kac, W. L. Murdock, and G. Szegö, On the eigenvalues of certain Hermitian forms, J. Rational Mech. Anal. vol. 2 (1953) pp. 767-800.

4. S. V. Parter, Extreme eigenvalues of Toeplitz forms and applications to elliptic difference equations, Trans. Amer. Math. Soc., to appear.

5. $\mathrm{H}$. Widom, On the eigenvalues of certain Hermitian operators, Trans. Amer. Math. Soc. vol. 88 (1958) pp. 491-522.

6. - Stable processes and integral equations, Trans. Amer. Math. Soc., to appear.

7. H. F. Weinberger, Lower bounds for higher eigenvalues by finite difference methods, Pacific J. Math. vol. 8 (1958) pp. 339-368.

Cornell University 